\title{
Les Collemboles des sables littoraux de la côte Ouest de Madagascar, plus spécialement ceux d'une plage de Mahajanga
}

\author{
The springtails of the littoral sands of Madagascar's western coast, \\ more specifically those from a beach at Mahajanga
}

\section{Ногохвостки миторальных песков западного берега Мадагаскара, точнее пмяжа у Махажканги}

\author{
Jean-Marc Thibaud \\ Ж.-М. Тибо
}

MECADEV, UMR 7179 CNRS, Muséum national d'Histoire naturelle, Sorbonne Universités, C.P. 50, 57 rue Cuvier, F-75231 Paris cedex 05, France. E-mail: thibaud@mnhn.fr

MOTS CLÉS: Collembola, Madagascar, sables littoraux, plage, variation annuelle, Mahajanga.

KEY WORDS: Collembola, Madagascar, littoral sands, beach, annual variation, Mahajanga.

КЛЮЧЕВЫЕ СЛОВА: Collembola, Мадагаскар, литоральные пески, пляж, годичная изменчивость, Махажанга.

RÉSUMÉ. Ce travail est un récapitulatif des espèces de Collemboles littoraux interstitiels connues de la côte Ouest de Madagascar où nous avons trouvé 15 espèces. Nous étudions ensuite les variations de la population de ces hexapodes d'une plage de Mahajanga, suivis tous les deux ans, de 2006 à 2018.

ABSTRACT. This work summarizes the current knowledge of terrestrial littoral sand species of Collembola from Madagascar's western coast, where 15 species have been found. It includes the population variations of this hexapod group in a beach north of Mahajanga sampled for two years round between 2006 and 2018.

РЕЗЮМЕ. Данная работа суммирует современные знания по наземным литоральным псаммобионтным видам ногохвосток с западного берега Мадагаскара, где отмечено 15 видов. Она включает и изменчивость популяций этой группы гексапод на пляже к северу от Махажанги, где два полных года между 2006 и 2018 брались пробы.

\section{Introduction}

Les Collemboles des sables littoraux sont assez mal connus surtout dans les zones tropicales ou subtropicales. Des études récentes ont été entreprises :

- en Amérique du Sud: aux Petites Antilles [Thibaud, Najt, 1992, Thibaud, 1993], à Cuba [Thibaud, 1994], au
Brésil [Mendonça, Fernandes, 1997; Thibaud, PalaciosVargas, 1999; Fernandes, Mendonça, 2002, 2004, 2007; Abrantes, Mendonça, 2007; D'Haese, Thibaud, 2011], au Mexique [Palacios-Vargas, Thibaud, 1998, 2001; Thibaud, Palacios-Vargas, 2000, 2001], au Venezuela [Thibaud, Diaz, 1998] et en Guyane Française [Thibaud, 2004].

- en Afrique: en Mauritanie [Thibaud, 1996], au Maroc [Potapov, Thibaud, 2003; Thibaud, Boumezzough, 2006, 2010], au Sénégal [Thibaud, Ndiaye, 2006], au Congo-Brazza [Thibaud, 2009c] et à Madagascar, Maurice, la Réunion, Mayotte, la Grande Comore, RodriguesMaurice [Thibaud, 2008a, b, 2010a, b, 2012, 2015, 2017].

- en Asie-Pacifique: en Corée du Sud [Thibaud, Lee, 1994; Thibaud, Kim, 1995], en Nouvelle-Calédonie [Thibaud, Weiner, 1997], au Vietnam [Thibaud, 2002, 2009a] et au Vanuatu [Thibaud, 2009b].

La présence de ces interstitiels terrestres est en partie liée à la granulométrie des sables littoraux, comprise entre $130 \mu \mathrm{m}$ et $1 \mathrm{~mm}$. Leur diversité est moins riche que celle des sols et des litières, car ces sols sableux sont trophiquement pauvres et supportent des facteurs climatiques (températures et humidité) très variables et très souvent drastiques [Thibaud, 2007].

Nous étudions ici la diversité des Collemboles littoraux interstitiels terrestres de la zone supralittorale, zone située au-dessus de la zone intercotidale, de la côte Ouest de Madagascar. Nous étudions ensuite la variation du peuplement en Collemboles d'une plage située au Village Touristique, au nord de la ville de Mahajanga et ce tous les deux ans, de 2006 à 2018.

How to cite this article: Thibaud J.M. 2020. Les Collemboles des sables littoraux de la côte Ouest de Madagascar, plus spécialement ceux d'une plage de Mahajanga // Russian Entomol. J. Vol.29. No.1. P.1 -5. doi: 10.15298/rusentj.29.1.01 


\section{Matériel et méthodes}

Nous avons pris en compte les espèces trouvées dans les sables littoraux de 5 localités de la côte Ouest, à savoir du nord au sud, Nosy Be (4 prélèvements), Mahajanga (17 prélèvements), Morondava-Kimany (4 prélèvements) et Toliara (Tuléar) ( 8 prélèvements : 15 $\mathrm{km}$ au sud et $30 \mathrm{~km}$ au nord). Chaque prélèvement étant constitué de 2 fois 2 litres de sable environ ( 2 fois, $20 \mathrm{~cm}$ x $20 \mathrm{~cm}$ x $5 \mathrm{~cm}$ d'épaisseur).

Pour le suivi tous les deux ans, pendant treize ans (2006-2018), à Mahajanga-Village Touristique, les prélèvements (deux devant l'hôtel « chez Karon » et deux au centre de la plage du Village Touristique) sont constitués chacun de 2 fois 2 litres de sable. Les dates de ces récoltes furent: 10/11.III.2006; 27/28.V.2008; 27/ 28.V.2010; 1/2.VI.2012; 12/13.VI.2014; 13/14.VI.2016; 22/24.X.2018, toutes effectuées le matin vers $9 \mathrm{~h}$.

La récolte des Collemboles se fait par « lavage de sable ». Le tri est réalisé de suite et les animaux sont conservés dans l'alcool. Ils sont ensuite, au laboratoire, éclaircis et montés entre lame et lamelle dans la solution de Marc André II, puis examinés au microscope à contraste de phase (x1000).

\section{Liste des stations}

A Mahajanga les sables sont assez divers, de moyens à grossiers (taille des grains comprise entre 150 et $600 \mu \mathrm{m}$, moyenne: $350 \mu \mathrm{m})$.

St. 1 - Mahajanga; sable au centre de la plage du GrandPavois. 09-03-2006.

St. 2 - Mahajanga; sable à l'entrée de la plage du Village Touristique, devant l'hôtel « chez Karon ». 10-03-2006.

St. 3 - Mahajanga; idem St. 2, mais un km plus au nord. 11-03-2006.

St. 4 - Mahajanga; idem St. 3, sable mouillé sous "Posidonies". 11-03-2006.

St. 5 - Mahajanga; idem St.3. 11-03-2006.

St. 6 - Mahajanga; sable de la plage du village touristique devant l'hôtel « chez Karon ». 27-05-2008.

St. 7 - Mahajanga; sable au centre de la plage du village touristique. 28-05-2008.

St. 8 - Mahajanga; sable de la plage du village touristique devant l'hôtel « chez Karon ». 27-05-2010.

St. 9 - Mahajanga; sable au centre de la plage du village touristique. 28-05-2010.

St. 10 - Mahajanga; sable de la plage du village touristique devant l'hôtel « chez Karon ». 01-06-2012.

St. 11 - Mahajanga; sable au centre de la plage du village touristique. 02-06-2012.

St. 12 - Mahajanga; sable de la plage du village touristique devant l'hôtel " chez Karon ». 12-06-2014.

St. 13 - Mahajanga; sable au centre de la plage du village touristique. 13-06-2014.

St. 14 - Mahajanga; sable de la plage du village touristique devant l'hôtel « chez Karon ». 13-06-2016.

St. 15 - Mahajanga; sable au centre de la plage du village touristique. 14-06-2016.

St.16 - Mahajanga; sable de la plage du village touristique devant l'hôtel « chez Karon ». 22-10-2018.
St. 17 - Mahajanga; sable au centre de la plage du village touristique, aux pieds de la digue. 24-10-2018.

\section{Systématique}

Famille Hypogastruridae Börner 1913

Genre Acherontiella Absolon, 1913

Acherontiella thibaudi Barra, 1994

MATERIEL ETUDIE. Mahajanga. Sts.3-4, 6, 7, 9, 10, 11, 13, 14, 16, 17. (cf. tableau page 4 ). - Morondave-Kimany: 6 ex. Tuléar: $15 \mathrm{~km}$ au sud, sable de la plage de Melody Beach: 15 ex.

Espèce tropicale, décrite de la République Sud-Africaine, connue du Vietnam [Thibaud, 2002], de Madagascar [Thibaud, 2008a], de Maurice [Thibaud, 2008b], de la Réunion [Thibaud, 2010a], de Mayotte [Thibaud, 2010b], de la Grande Comore [Thibaud, 2012] et de Rodrigues-Maurice [Thibaud, 2017].

Espèce psammobionte, très commune. Elle est connue à Madagascar de plages à Mahajanga, Morondave-Kimany, Tuléar et dans 9 stations de la côte-Est, ainsi que dans toutes les îles limitrophes étudiées de l'Océan Indien et ce, souvent, en grand nombre d'individus.

Genre Xenylla Tullberg, 1869

Xenylla yucatana Mills, 1938

MATERIEL ETUDIE. Mahajanga. Sts. 1: 22 ex.; 2, 3-4, 6, 7, 8, $9,10,11,12,13,14,16,17$. (cf. tableau page 4 ).

Espèce pantropicale, décrite du Mexique et retrouvée dans les zones néotropicale, afrotropicale, orientale et récemment du Yemen [Barra, 2006], à Madagascar [Thibaud, 2008a], au Vanuatu [Thibaud, 2009b] et à Rodrigues-Maurice [Thibaud, 2017].

Espèce édaphique, guanophile et psammophile, assez rare. Signalée à Madagascar de plages à Mahajanga, île SteMarie et Vatomandry.

Famille Neanuridae Börner, 1901

Frieseinae Massoud, 1967

Genre Friesea Dalla Torre, 1895

Friesea mahajanga Thibaud, 2008

MATERIEL ETUDIE. Mahajanga. Sts. 2, 3-4, 7, 8, 9, 10, 11, 13, 14, 16. (cf. tableau page 4). - Nosy Be: sable de la plage d'Ambaro: 1 ex.

Espèce décrite de Madagascar, retrouvée à Maurice [Thibaud, 2008a, b] et à la Réunion [Thibaud, 2010a].

Espèce psammobionte, assez rare. Elle est connue à Madagascar de plages à Mahajanga, Nosy Be, île Ste-Marie, Raména et Sambava.

Friesea petiti (Delamare Deboutteville et Massoud, 1964)

MATERIEL ETUDIE. Mahajanga. Sts. 3-4, 6, 7, 8, 9, 10, 11,

$12,13,14,16,17$. (cf. tableau page 4 )

Espèce endémique de Madagascar [Delamare Deboutteville, Massoud, 1964; Thibaud, 2008a].

Espèce psammobionte, assez rare. Elle est connue à Madagascar de plages à Fort Dauphin et Mahajanga.

Famille Odontellidae Massoud, 1967

Genre Odontellina Deharveng, 1981

Odontellina deharvengi Barra, 1995

MATERIEL ETUDIE. Tuléar, $20 \mathrm{~km}$ au sud, plage de Sarodrano. 1 ex. 
Espèce décrite des sables littoraux de la Province du Natal en République Sud-Africaine.

Espèce psammobionte, rare. Elle n'est connue à Madagascar que d'une plage au sud de Tuléar.

Famille Tullbergiidae Bagnall, 1935

(sensu Deharveng, 2004)

Genre Fissuraphorura Rusek, 1991

Fissuraphorura cubanica Rusek, 1991

MATERIEL ETUDIE. Mahajanga. Sts. 3-4, 7, 8, 9, 10, 11, 13, 14, 16. (cf. tableau page 4).

Espèce pantropicale, décrite de Cuba, retrouvée au Nicaragua, au Vietnam, en Guyane Française, à Madagascar [Thibaud, 2008a], au Vanuatu [Thibaud, 2009b] et à la Réunion [Thibaud, 2010a].

Espèce hémiédaphique-psammophile, assez commune. Elle est connue à Madagascar de plages à Mahajanga, île Ste-Marie, Vatomandry, Sambava, Antalaha, Tamatave et Mahambo.

Genre Spicatella Thibaud, 2002

Spicatella cf. bedosae Thibaud, 2002

MATERIEL ETUDIE. Mahajanga. Sts. 3-4, 6, 8, 10, 12, 13, 14. (cf. tableau page 4 ).

Espèce décrite du Vietnam, retrouvée à Madagascar [Thibaud, 2008a],

Espèce psammobionte. Elle n'est connue à Madagascar que d'une plage à Mahajanga,

Famille Isotogastruridae Thibaud et Najt, 1992

Genre Isotogastrura Thibaud et Najt, 1992

Isotogastrura madagascariensis Thibaud, 2008

MATERIEL ETUDIE. Tuléar, $30 \mathrm{~km}$ au nord, sable de la plage d'Ifaty: 18 ex.

Espèce endémique de Madagascar, décrite d'une plage de Fort Dauphin [Thibaud, 2008a].

Espèce psammobionte, assez rare. Elle est connue à Madagascar de plages à Fort Dauphin, Tuléar-Ifaty et Antalaha.

Famille Isotomidae Schaeffer, 1896

Genre Archisotoma Linnaniemi, 1912

Archisotoma madagascariensis Thibaud, 2008

MATERIEL ETUDIE. Nosy Be, sable de la plage d'Ambaro: 1 ex. Espèce décrite d'une plage de Fort Dauphin [Thibaud, 2008a], retrouvée à Maurice [Thibaud, 2008b] et à la Grande Comore [Thibaud, 2012].

Espèce psammobionte, assez rare. Elle est connue à Madagascar de plages à Nosy Be, Fort Dauphin, Tamatave et Antalaha.

Archisotoma vaoensis Thibaud et Weiner, 1997

MATÉRIEL ÉTUDIÉ. Mahajanga. Sts. 1: 2 ex., 3-4, 7, 8, 9, 10, 12, 13, 16. (cf. tableau page 4 ).

Espèce décrite de Nouvelle-Calédonie, retrouvée au Vietnam, à Madagascar [Thibaud, 2008a], à Maurice [Thibaud, 2008b], au Vanuatu [Thibaud, 2009b] et à la Grande Comore [Thibaud, 2012].

Espèce psammobionte, assez rare. Elle est connue à Madagascar de plages à Mahajanga, Fort Dauphin, île SteMarie, Antalaha et Manakara.

Genre Folsomides Stach, 1922

Folsomides parvulus Stach, 1922

MATERIEL ETUDIE. Mahajanga. Sts. 16, 17. (cf. tableau page
Espèce décrite de Hongrie, cosmopolite, très commune sous les tropiques, présente à Madagascar [Thibaud, 2008a, 2015] et à Maurice [Thibaud, 2008b].

Espèce hémiédaphique-guanophile et psammophile. Espèce assez rare dans les sables littoraux de Madagascar. Elle est connue de plages à île Ste-Marie, Raména, Vohémar, Manakara et Mahajanga.

Genre Folsomina Denis 1931

Folsomina onychiurina Denis, 1931

MATERIEL ETUDIE. Mahajanga. Sts. 5: 1 ex. 6, 7, 9, 10, 11, $12,13,14,16$. (cf. tableau page 4 ).

Espèce à large répartition décrite du Costa Rica, déjà signalée en Afrique du Sud [Barra, 1997], à Madagascar [Thibaud, 2008a], à Maurice [Thibaud, 2008b], à la Réunion [Thibaud, 2010a], à Mayotte [Thibaud, 2010b], à la Grande Comore [Thibaud, 2012] et à Rodrigues-Maurice [Thibaud, 2017].

Espèce euédaphique-psammophile la plus commune des plages de Madagascar et récoltée souvent en grand nombre d'individus. Elle est ainsi connue de plages à Mahajanga, Fort Dauphin, île Ste-Marie, Vatomandry, Raména, Vohémar, Sambava, Antalaha, Manakara, Tamatave, Fénérive et Mahambo.

\section{Genre Hemisotoma Bagnall 1949}

Hemisotoma thermophila (Axelson, 1900)

MATERIEL ETUDIE. Mahajanga. St. $1: 6$ ex., 2, 3-4, 6, 7, 8, $9,10,11,12,13,14,16,17$. (cf. tableau page 4 ) — Nosy Be: sable de la plage d'Andilana: 2 ex.

Espèce cosmopolite, trouvée aussi à Madagascar [Thibaud, 2008a], à Maurice [Thibaud, 2008b] et à la Grande Comore [Thibaud, 2012].

Espèce édaphique-psammophile, assez commune à Madagascar où elle est connue de plages à Mahajanga, Nosy Be, île Ste-Marie, Vatomandry, Raména, Sambava, Antalaha et une entre Foulpointe et Tamatave.

Genre Pauropygus Potapov, Gao et Deharveng 2013

Pauropygus caussaneli (Thibaud, 1996)

MATERIEL ETUDIE. Mahajanga. Sts. 3-4, 6, 7, 8, 9, 10, 11, $12,13,14,16,17$. (cf. tableau page 4 ). - Tuléar: $20 \mathrm{~km}$ au sud, sable de la plage de Sarodrano: 3 ex. et $30 \mathrm{~km}$ au nord, sable de la plage d'Ifaty: 2 ex.

Espèce décrite de Mauritanie, retrouvée en Afrique du Sud [Barra, 1997], au Sénégal, au Maroc, à Madagascar [Thibaud, 2008a], à Maurice [Thibaud, 2008b], à Mayotte [Thibaud, 2010b], à la Grande Comore [Thibaud, 2012] et à Rodrigues-Maurice [Thibaud, 2017].

Espèce psammobionte, assez commune à Madagascar où elle est connue de plages à Mahajanga, Tuléar, Fort Dauphin, île Ste-Marie, Vohémar, Sambava, Antalaha et une entre Foulpointe et Tamatave.

Genre Psammisotoma Greenslade et Deharveng, 1986

Psammisotoma kingae Greenslade et Deharveng, 1986

MATERIEL ETUDIE. Mahajanga. St. 1: 7 ex, 2, 3-4, 7, 8, 9, $10,11,12,13,14,16$. (cf. tableau page 4).

Espèce décrite d'Australie, retrouvée en Papouasie-Nouvelle-Guinée, en Indonésie, en Nouvelle-Calédonie, au Sénégal et à Madagascar [Thibaud, 2008a].

Espèce psammobionte, intertidale et supralittorale, assez rare. A Madagascar elle est connue de plages à Mahajanga, Fort Dauphin et île Ste-Marie. 
Table. Données sur la variation du peuplement en Collemboles d'une plage de Mahajanga (pendant 13 ans: 2006-2018). Таблица. Данные по изменчивости населения коллембол пляжа у Махажанги в течение 13 лет (2006-2018).

\begin{tabular}{|l|c|c|c|c|c|c|c|c|c|c|c|c|c|c}
\hline \multicolumn{1}{|c|}{} & 2006 & 2008 & 2010 & 2012 & 2014 & 2016 & 2018 \\
\hline Stations (plages) & 2 & $3 / 4$ & 6 & 7 & 8 & 9 & 10 & 11 & 12 & 13 & 14 & 15 & 16 & 17 \\
\hline Acherontiella.thibaudi & 0 & 1 & 1 & 1 & 0 & 1 & 2 & 1 & 0 & 2 & 1 & 0 & 1 & 1 \\
\hline Xenylla yucatana & 12 & 4 & 10 & 6 & 14 & 8 & 10 & 10 & 16 & 10 & 15 & 0 & 13 & 2 \\
\hline Friesea mahajanga & 3 & 1 & 0 & 1 & 2 & 1 & 5 & 2 & 0 & 2 & 3 & 0 & 2 & 0 \\
\hline Friesea petiti & 0 & 2 & 1 & 2 & 2 & 5 & 6 & 2 & 6 & 1 & 4 & 0 & 8 & 1 \\
\hline Fissuraphorura.cubanica & 0 & 3 & 0 & 2 & 2 & 2 & 3 & 2 & 0 & 4 & 2 & 0 & 2 & 0 \\
\hline Spicathella cf. bedosae & 0 & 20 & 2 & 0 & 1 & 0 & 5 & 0 & 1 & 2 & 1 & 0 & 0 & 0 \\
\hline Archisotoma vaoensis & 0 & 1 & 0 & 1 & 1 & 1 & 2 & 0 & 1 & 1 & 0 & 0 & 4 & 0 \\
\hline Folsomides parvulus & 0 & 0 & 0 & 0 & 0 & 0 & 0 & 0 & 0 & 0 & 0 & 0 & 1 & 3 \\
\hline Folsomina onychiurina & 0 & 0 & 1 & 1 & 0 & 1 & 2 & 1 & 2 & 1 & 1 & 0 & 1 & 0 \\
\hline Hemisotoma thermophila & 2 & 1 & 6 & 6 & 7 & 5 & 12 & 8 & 16 & 6 & 8 & 0 & 13 & 6 \\
\hline Pauropygus caussaneli & 0 & 6 & 2 & 5 & 3 & 4 & 2 & 5 & 3 & 6 & 6 & 0 & 1 & 1 \\
\hline Psammisotoma kingae & 3 & 7 & 0 & 1 & 1 & 1 & 3 & 2 & 5 & 8 & 4 & 0 & 2 & 0 \\
\hline total & 20 & 46 & 23 & 26 & 33 & 29 & 52 & 33 & 50 & 43 & 45 & 0 & 48 & 14 \\
\hline
\end{tabular}

Signalons que les travaux de confection d'une digue pour protéger la côte et le Village Touristique à $3 \mathrm{~km}$ au nord de la ville de Mahajanga en 2016-2017, ont fait disparaitre la station située au centre de la plage du Village Touristique pendant un certain temps. Celle-ci très bouleversée, s'est un peu reconstituée en 2018, avec quelques touffes éparses de végétation. Par contre, la plage en face de l'hôtel « chez Karon »n'a été que peu touchée et la végétation a été, en partie, préservée.

\section{Conclusion}

Le nombre total d'espèces de Collemboles interstitiels terrestres trouvées dans la zone sableuse sublittorale de la côte Ouest de Madagascar est de 15, ceci dans les 5 localités étudiées. Rappelons que pour 12 localités étudiées sur la côte Est le nombre d'espèces connues est de 30. Les espèces communes aux deux côtes, est et ouest, sont au nombre de 13. Ce qui fait un total de 34 espèces interstitielles sableuses connues des côtes de Madagascar.

Ajoutons, qu'une espèce endémique de NeanuridaePseudachorutinae, Oudemansia dubia Denis, 1947, a été décrite d'un récif corallien du Cap d'Ambre à l'extrême pointe nord de l'île.

Pour la partie ouest, la localité la plus riche, mais aussi la plus étudiée, est la plage du Village Touristique de Mahajanga (12 espèces/15), puis celles de Tuléar (4) et de Nosy Be (3).

L'espèce la plus commune le long de cette côte ouest serait l'Hypogastruridae Xenylla yucatana, ceci par sa présence dominante à Mahajanga.

Dans le suivi de la plage du Village Touristique de Mahajanga, suivi tous les deux ans pendant 13 ans, le nombre d'espèces par stations varie de 0 (pour la station 15 en 2016, à cause des travaux de la construction de la digue) à 11 , avec une moyenne de 8 espèces. Le nombre d'individus varie de 0 à 52 , avec une moyenne de 33 individus. Pour la côte est, il en était, respectivement, de 2 à 16 (moyenne de 4,5 espèces) et de 6 à 150 (moyenne de 36 individus).
Comme dans notre étude sur deux ans de la plage d'Antalaha [Thibaud, 2015], le nombre d'espèces et d'individus semblent assez constant, indépendamment du problème des travaux dus à la construction de la digue protectrice de la côte. Ceci montrerait une relative stabilité des populations de Collemboles interstitiels terrestres étudiés sur une assez longue période. Il en est, bien évidemment, tout autre dans les écosystèmes perturbés ou détruits par les travaux d'aménagement et de bétonnage de la côte.

Rappelons que les espèces psammobiontes sont morphologiquement proches des espèces euédaphiques. Elles sont en effet courtes (0,3 à 0,7 mm), dépigmentées, micro- ou anophtalmes, montrent des pattes courtes, une furca réduite ou absente. Leur corps est flexible pour pouvoir s'insérer entre les grains de sable sans en bouleverser la structure.

REMERCIEMENTS. Je remercie bien vivement Sergei Golovatch (Moscou, Russie) pour l'aide apportée à la publication de cette note.

\section{Références}

Abrantes E.A., Mendonça Cl. de. 2007. New species and a new record of Isotomidae (Collembola) from the coast of Brazil // Zootaxa. Vol.1500. P.55-60.

Axelson W.M. 1900. Vorläufige Mittheilung über einige neue Collembolen-Formen aus Finland // Meddelanden af Societas pro Fauna et Flora Fennica. Bd.26. S.1-19.

Barra J.-A. 1994. Nouveaux collemboles Poduromorphes de la Province du Natal (Rép. Sud Africaine) (Insecta: Collembola) // Journal of African Zoology. Vol.108. P.181-189.

Barra J.-A. 1995. Nouveaux collemboles Poduromorphes des sables littoraux (partie terrestre) de la province du Natal (Rép. Sud Africaine) (Insecta: Collembola) // Journal of African Zoology. Vol.109. P.125-139.

Barra J.-A. 1997. Nouveaux collemboles Entomobryomorphes des sables littoraux (partie terrestre) de la Province du Natal (Rép. Sud Africaine) (Insecta: Collembola) // Journal of African Zoology. Vol.111. P.465-480.

Barra J.-A. 2006. Collemboles de 1'île de Socotra, République du Yemen // Zoosystema. T.28. P. 61-74. 
Delamare Deboutteville C., Massoud Z. 1964. Collemboles marins interstitiels des plages de Madagascar// Vie et Milieu. Suppl.17. P.381-392.

Denis J.R. 1931. Contributo alla conoscenza del "Microgenton" di Costa Rica // Bollettino del Laboratorio di Zoologia generale ed agraria del R. Istituto superiore agrario di Portici. Vol.25. P.69-170.

D'Haese C.A., Thibaud J.-M. 2011. Description and phylogenetic position of a new Willemia species (Collembola Hypogastruridae) from the littoral coast of Brazil // Zootaxa. Vol.2932. P.33-40.

Fernandes L.H., Mendonça M.Cl. de. 2002. Duas novas espécies de Pseudachorutinae (Collembola, Neanuridae) do Brasil // Boletim do Museu Nacional, Rio de Janeiro. No.496. P.1-8.

Fernandes L.H., Mendonça M.Cl. de. 2004. Collembola Poduroporpha de litoral de Marica, Rio de Janeiro, Brasil // Revista Brasileira de Zoologia. Vol.21. P.15-25.

Fernandes L.H., Mendonça M.Cl. de. 2007. Collembola Poduromorpha de àreas preservadas e impactadas do litoral sudeste do Brasil // Revista Brasileira de Zoologia. Vol.24. P.777-785.

Grennslade P., Deharveng L. 1986. Psammisotoma, a new genus of Isotomidae (Collembola) from marine littoral habitats // Proceedings of the Royal Society of Queensland. Vol.97. P.89-95.

Mendonça M.Cl. de, Fernandes L.H. 1997. A new genus of Brachystomellinae from Brazil (Collembola: Neanuridae) // Boletin do Museu Nacional, Rio de Janeiro. No.379. P.1-7.

Mills H.B. 1938. Collembola from Yucatan caves // Carnegie Institution of Washington Publications. Vol.491. P. 183-190.

Palacios-Vargas J.G., Thibaud J.-M. 1998. Two new Mexican Isotogastrura (Collembola: Isotogastruridae) // Canadian Entomologist. Vol.130. P.195-199.

Palacios-Vargas J.G., Thibaud J.-M. 2001. Three new species of Mexican littoral Collembola of genera Willemia, Cryptopygus and Isotogastrura (Hypogastruridae, Isotomidae, Isotogastruridae) // Revue française d'Entomologie. Vol.23. P.161-168.

Potapov M., Thibaud J.-M. 2003. Collemboles interstitiels des sables littoraux et continentaux du Maroc (Collembola) // Revue française d'Entomologie. Vol.25. P.117-122.

Rusek J. 1991. New tropical Tullbergiinae (Collembola: Onychiuridae) // Acta Entomologica Bohemoslovaca. Vol.88. P.145-155.

Thibaud J.-M. 1993. Les Collemboles des Petites Antilles. VI Interstitiels terrestres et marins // Revue française d'Entomologie. Vol.15. P.69-80.

Thibaud J.-M. 1994. Les Collemboles interstitiels terrestres de l'île de Cuba, avec la description de deux espèces nouvelles // Revue française d'Entomologie. Vol.16. P.93-98.

Thibaud J.-M. 1996. Étude des collemboles (Hexapoda) interstitiels des sables littoraux de Mauritanie // Annales de la Société entomologique de France. Vol.32. P.475-479.

Thibaud J.-M. 2002. Contribution à la connaissance des Collemboles interstitiels des sables littoraux du Vietnam //Revue française d'Entomologie. Vol.24. P.201-209.

Thibaud J.-M. 2004. Collemboles interstitiels des sables littoraux de Guyane Française (Collembola) // Revue française d'Entomologie. Vol.26. P.63-66.

Thibaud J.-M. 2007. Recent advances and synthesis in biodiversity and biogeography of arenicolous Collembola // Annales de la Société entomologique de France. Vol.43. P.181-185.

Thibaud J.-M. 2008a. Les collemboles des sables littoraux de Madagascar// Annales de la Société entomologique de France. Vol.44. P.503-519.
Thibaud J.-M. 2008b. Les collemboles des sables littoraux de l'île Maurice // Revue française d'Entomologie. Vol.30. P.71-76.

Thibaud J.-M. 2009a. Contribution à la connaissance des Collemboles interstitiels des sables littoraux du Vietnam (suite) // Revue française d'Entomologie. Vol.31. P.33-36.

Thibaud J.-M. 2009b. Les collemboles (Collembola) interstitiels des sables littoraux de l'île d'Espiritu Santo (Vanuatu) // Zoosystema. Vol.31. No.3. P.499-505.

Thibaud J.-M. 2009c. Les Collemboles du littoral Congolais // Revue française d'Entomologie. Vol.31. P.131-134.

Thibaud J.-M. 2010a. Contribution à la connaissance des collemboles littoraux de l'île de la Réunion // Revue française d'Entomologie. Vol.32. P.25-31.

Thibaud J.-M. 2010b. Les collemboles des sables littoraux de l'île de Mayotte. Essai de synthèse sur les collemboles des sables littoraux d'îles de l'océan Indien (zone ouest) // Revue française d'Entomologie. Vol.32. P.113-121.

Thibaud J.-M. 2012. Les collemboles des sables littoraux de l'île de la Grande Comore // Revue française d'Entomologie. Vol.33. P.5-12.

Thibaud J.-M. 2015. Etude des Collemboles des sables littoraux de la côte Est de Madagascar (interstitiels terrestres) // Zoosystema. Vol.37. P.73-84.

Thibaud J.-M. 2017. Les Collemboles interstitiels des sables littoraux de l'île Rodrigues (île Maurice) // Russian Entomological Journal. Vol.26. P.299-301.

Thibaud J.-M., Boumezzough A. 2006. Collemboles interstitiels des sables littoraux du Maroc - II // Revue française d'Entomologie. Vol.28. P.63-67.

Thibaud J.-M., Boumezzough A. 2010. Sur quelques Collemboles des sables des côtes marocaines - III. Liste des espèces connues du Maroc // Revue française d'Entomologie. Vol.32. P.177-180.

Thibaud J.-M., Diaz A. 1998.Collemboles interstitiels de sables continentaux et fluviatiles du Venezuela // Zoosystema. Vol.20. P.123-127.

Thibaud J.-M., Kim J.T. 1995. One new species of Collembola (Insecta) from sand dunes of Korea // Korean Journal of Systematic Zoology. Vol.11. P.479-484.

Thibaud J.-M., Lee B.-H. 1994. Three new species of interstitial Collembola from sand dunes of South Korea // Korean Journal of Systematic Zoology. Vol.10. P.39-46.

Thibaud J.-M., Najt J. 1992. Isotogastruridae, a new family of terrestrial interstitial Collembola from the Lesser Antilles // Bonner zoologische Beiträge. Vol.43. P.545-551.

Thibaud J.-M., Ndiaye A.B. 2006. Collemboles interstitiels des sables littoraux du Sénégal // Revue française d'Entomologie. Vol.28. P.41-48.

Thibaud J.-M., Palacios-Vargas J.G. 1999. Brazilian Collembolla from littoral sand with description of Austrogastrura gen. n. and Isotomodes carioca sp. n. (Hypogastruridae; Isotomidae) // Revue française d'Entomologie. Vol.21. P.25-31.

Thibaud J.-M., Palacios-Vargas J.G. 2000. Une nouvelle espèce du genre Mesaphorura des sables littoraux du Mexique (Collembola, Onychiuridae) // Revue française d'Entomologie. Vol.22. P.146-148.

Thibaud J.-M., Palacios-Vargas J.G. 2001. Collemboles interstitiels des sables littoraux du Mexique (Collembola) // Revue française d'Entomologie. Vol. 23. P.181-184.

Thibaud J.-M., Weiner W.M. 1997. Collemboles interstitiels des sables de Nouvelle Calédonie // Mémoires du Muséum national d'Histoire naturelle. Vol.171. P.63-89. 